\title{
An Uncommon Case of Ectopic Pregnancy: Cornual Pregnancy after Salpingectomy
}

\author{
Melhaoui Houda ${ }^{*}$, Nassim Kaoutar, Rachid Bezad, Alami MH \\ Motherhood of orange trees, CNSR flap, Mororocco
}

DOI: $10.36348 /$ sijog.2020.v03i02.008

| Received: 08.02.2020 | Accepted: 15.02.2020 | Published: 16.02.2020

*Corresponding author: Melhaoui Houda

\section{Abstract}

This is a case report describing an ectopic pregnancy occurring in the remnant stump following salpingectomy. A pregnancy implanted in this location appears very uncommon, thereby rendering the correct diagnosis challenging. This contribution comprises a case description, a presentation of the different diagnostic and therapeutic methods available to date, as well as a succinct literature review. We have also highlighted the requirement of performing salpingectomy by sectioning the isthmus of the uterine tube at the uterine horn, then coagulating the horn's intramural portion. Lastly, we have taken into consideration a series of practical recommendations.

Keywords: Interstitial pregnancy, methotrexate, salpingectomy, laparoscopy.

Copyright @ 2020: This is an open-access article distributed under the terms of the Creative Commons Attribution license which permits unrestricted use, distribution, and reproduction in any medium for non-commercial use (NonCommercial, or CC-BY-NC) provided the original author and source are credited.

\section{Patient ANd OBSERVATion}

A 29-year-old female patient presented to the emergency room, complaining of the sudden onset of pain in the left iliac fossa and hypogastrium. This patient has a heavy obstetric history, she is G3P1. In this case, she had a spontaneous and normal pregnancy with a vaginal delivery. A year later, she had a tubal ectopic pregnancy treated by right salpingectomy. A few years after this episode and faced with a secondary sterility table, with no etiology highlighted, the patient and her partner begin cycles of in vitro fertilization (IVF). The patient is then spontaneously pregnant. Given the date of her last period, the patient is 4 weeks pregnant with amenorrhea on the day she comes to the emergency room. The emergency blood test shows an $\mathrm{HCG}$ at $5035 \mathrm{mU} / \mathrm{ml}$. The patient initially experienced an exacerbation of her pain in the left iliac fossa. Consequently, an endovaginal ultrasound is performed to observe a lateral uterus deviated to the left, not containing an intrauterine gestational sac, the Douglas cul-de-sac is cluttered with a poorly delimited and heterogeneous mass reminiscent of clots

In view of the patient's history, the ultrasound aspect and especially her clinical and hemodynamic state, we decide to perform a laparotomy
When the peritoneum opens, we see a peritoneal cavity filled with clotted blood. After rinsing and aspirating the Douglas cul-de-sac, we analyze the left appendix, which seems intact. On the right, we notice that the uterine angle is perforated and that blood is actively flowing through this opening. Using forceps, we extract a tissue mass resembling trophoblastic material from the uterine perforation. The extraction of pregnancy results in an immediate decrease in bleeding (Figure 1).

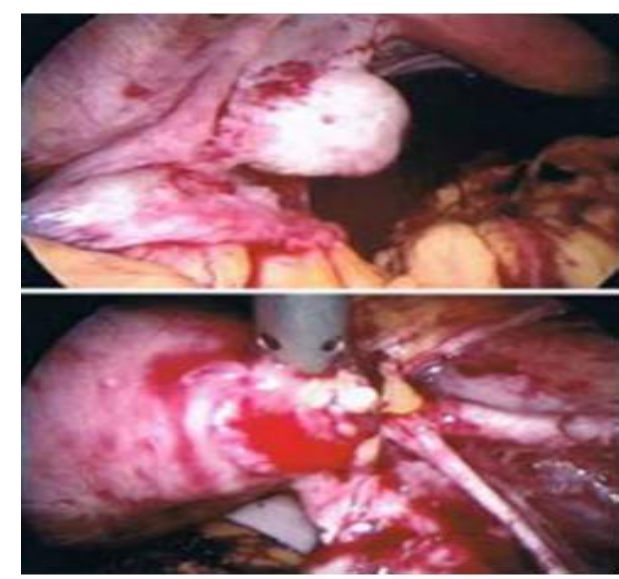

Fig-1: Corneal rupture dt 
We closed the uterine cavity using three $\mathrm{X}$ shaped points of vicryl 2-0, The post-operative consequences were marked by a transfusion of two units of blood in view of the clinical condition of the patient and her hemoglobin level ( $\mathrm{Hb} 6 \mathrm{~g} / \mathrm{dl}$ coming from $13.6 \mathrm{~g} / \mathrm{dl}$ ). She left the hospital four days after the surgery. To assess the risk of recurrence, hysterosalpingography is performed three months postoperatively. This shows a silent intra-mural portion on the right, which makes the risk of homolateral recurrence very low (Figure 2).

\section{LITERATURE REVIEW AND DISCUSSION}

The interstitial portion of the fallopian tube corresponds to the proximal segment incorporated into the uterine muscle wall $(0.7 \times 1.5 \mathrm{~cm})$. A pregnancy implanted there is called an interstitial pregnancy [2]. When it is located in a rudimentary horn of a bicornuate uterus or in the remaining stump of a fallopian tube treated by salpingectomy, it is called corneal pregnancy [21]. A review of the literature confirms the extremely rare nature of this location for an ectopic pregnancy. In fact, almost all (98\%) of ectopic pregnancies occur in the fallopian tubes [1]. The frequency of interstitial pregnancies is between $2-3 \%$ of all extrauterine pregnancies, with a mortality rate twice as high as tubal pregnancies [2, 22]. Interstitial, angular ectopic pregnancies (developed at the level of the tubal ostium at the bottom of the uterine cavity) and cornuals are often grouped together and represent the same clinical and therapeutic entity [21]. The rupture of interstitial pregnancies is particularly haemorrhagic due to a rich corneal vascularization and a greater myometrial distension by a more advanced pregnancy [19]. Interstitial pregnancies can be misdiagnosed as intrauterine due to their partial implantation in the endometrium. Contrary to popular belief, the rupture of interstitial pregnancies appears relatively early in pregnancy. The risk factors are similar to other ectopic pregnancies (history of ectopic pregnancy, pathology and tubal surgery, intrauterine exposure of DES, genital infections, tobacco, IVF) except for ipsilateral salpingectomy which is a factor of risk specific to interstitial pregnancy [3]. The diagnosis is based on a summary of the clinic, plasma HCG and transvaginal ultrasound [21]. The following ultrasound criteria were proposed by Timor-Tritsch in 1992 for this diagnosis: an empty uterine cavity, an eccentric gestational sac located $>1 \mathrm{~cm}$ from the lateral wall of the uterine cavity and a thin.

Outside of a context of rupture, it is lawful to offer patients medical treatment. There is no consensus regarding the limiting level of HCG or the presence of cardiac activity. Treatment by local route seems to obtain better results compared to MTX by general route. If the bag grows under MTX, surgical treatment is essential [21]. The mean duration of undetectable HCG level in serum is $43+/-64$ days [13]. An interstitial residual mass or a heterogeneous area with persistent vascularization on ultrasound has been reported [17]. Subject to complete negativation of HCG, they do not constitute a therapeutic failure [21]. Close monitoring of medically treated patients is advised. After medical treatment for an interstitial pregnancy, the risk of uterine rupture remains unknown for a future pregnancy [18]. This concern exists for interstitial pregnancies that are treated surgically and medically [8]. Given the fact that these techniques are only described for isolated cases, no data is available as to the solidity of the corneal scar during a subsequent intrauterine pregnancy. However, the operated uterine horn appears to be a fragile area and cases of rupture in the second trimester have been described. It is assumed that even after medical treatment, doubt persists on the quality of the corneal myometrium after treatment. Currently, most authors recommend performing a caesarean section before the onset of labor in a subsequent pregnancy [21]. Note that during a salpingectomy, it is important to perform a tubal section close to the uterus. Indeed, recurrences of ectopic pregnancies have been described in cases of residual tubal stump after salpingectomy [20].

In this case, the patient is suffering from a corneal pregnancy. Consequently, for this particular localization, we will advise against treatment with Metothrexate, and we will encourage practitioners to favor the surgical route. The therapeutic gesture being more complicated than the treatment of a classic ectopic pregnancy, gynecologists should not practice laparotomy (too invasive gesture) but rather learn the technique by coeliscopy in order to offer their patients optimal care. with regard to the clinical picture.

\section{PRACTICAL RECOMMENDATIONS}

- Interstitial pregnancy is a rare location $(2.4 \%)$ of ectopic pregnancy.

- This should be taken into account during an eccentric intrauterine location more than one centimeter from the lateral wall of the cavity, with a thin myometrial periphery.

- The reference treatment is surgical laparoscopy (in the absence of hemodynamic disorder). 4. Medical treatment can also be used in selected cases (without consensus on the eligibility criteria)

- It is important to follow the decay of the HCG until its negativation.

- A history of homolateral salpingectomy is a significant risk factor

\section{REFFERENCE}

1. Bouyer, J., Coste, J., Fernandez, H., Pouly, J. L., \& Job-Spira, N. (2002). Sites of ectopic pregnancy: a 10 year population-based study of 1800 cases. Human reproduction, 17(12), 3224-3230.

2. Tulandi, T. (2013). Incidence, risk factors, and pathology of ectopic pregnancy. Uptodate. Last update jul 30. 
Melhaoui Houda et al; Sch Int J Obstet Gynec, Feb. 2020; 3(2): 51-53

3. Tulandi, T., \& Al-Jaroudi, D. (2004). Interstitial pregnancy: results generated from the Society of Reproductive Surgeons Registry. Obstetrics \& Gynecology, 103(1), 47-50.

4. Timor-Tritsch, I. E., Monteagudo, A., Matera, C., \& Veit, C. R. (1992). Sonographic evolution of cornual pregnancies treated without surgery. Obstetrics and gynecology, 79(6), 10441049.

5. Filhastre, M., Dechaud, H., Lesnik, A., \& Taourel, P. (2005). Interstitial pregnancy: role of MRI. European radiology, 15(1), 93-95.

6. Júnior, E. A., Zanforlin Filho, S. M., Pires, C. R., Guimarães Filho, H. A., Massaguer, A. A., Nardozza, L. M. M., \& Moron, A. F. (2007). Three-dimensional transvaginal sonographic diagnosis of early and asymptomatic interstitial pregnancy. Archives of gynecology and obstetrics, 275(3), 207-210.

7. Moawad, N. S., Mahajan, S. T., Moniz, M. H., Taylor, S. E., \& Hurd, W. W. (2010). Current diagnosis and treatment of interstitial pregnancy. American journal of obstetrics and gynecology, 202(1), 15-29.

8. Warda, H., Mamik, M. M., Ashraf, M., \& Abuzeid, M. I. (2014). Interstitial ectopic pregnancy: conservative surgical management. JSLS: Journal of the Society of Laparoendoscopic Surgeons, 18(2), 197.

9. Tulandi, T., \& Al-Jaroudi, D. (2004). Interstitial pregnancy: results generated from the Society of Reproductive Surgeons Registry. Obstetrics \& Gynecology, 103(1), 47-50.

10. Soriano, D., Vicus, D., Mashiach, R., Schiff, E., Seidman, D., \& Goldenberg, M. (2008). Laparoscopic treatment of cornual pregnancy: a series of 20 consecutive cases. Fertility and sterility, 90(3), 839-843.

11. Meyer, W. R., \& Mitchell, D. E. (1989). Hysteroscopic removal of an interstitial ectopic gestation. A case report. The Journal of reproductive medicine, 34(11), 928-929.

12. Stovall, T. G., Ling, F. W., \& Buster, J. E. (1989). Outpatient chemotherapy of unruptured ectopic pregnancy. Fertility and sterility, 51(3), 435-438.

13. Lau, S. (2000). Conservative medical and surgical management of interstitial ectopic pregnancy. Fertil Steril, 73, 1063-1064.

14. Jermy, K., Thomas, J., Doo, A., Bourne, T. (2004). The conserva - tive management of interstitial pregnancy. BJOG, 111:1283.

15. Madelenat, P. (2003). Recommandations pour la pratique clinique. Prise en charge de la grossesse extra-uté - rine. CNGOF, Paris.

16. Tulandi, T. (2014). Methotrexate treatment of tubal and interstitial ectopic pregnancy. Uptodate. Jul

17. Tang, A., Baartz, D., Khoo, S.K. (2006). A medical management of interstitial ectopic pregnancy : a 5year clinical study. Aust N Z J Obstet Gynaecol, 46:107.

18. Ophir, E., Singer-Jordan, J., Oettinger, M.(2004). Uterine artery embolization for management of interstitial twin ectopic pregnancy : case report. Hum Reprod, 19:1774.

19. Malek-mellouli, M., Youssef, A., Mbarki, M., Ben, Amara, F., Néji, K., Reziga, H. (2012). Traitement médical des gros - sesses interstitielles non rompues. La Tunisie Medi - cale, 90 05) : 421 423.

20. Merviel, P., Lourde, E., Gagneur, O., Nasreddine, A., Brzakowski, M., Urrutiaguer, S. (2010). Grossesse extrautérines. Traitement chirurgical : techniques, avantages et inconvénients. CNGOF, Paris.

21. Jourdain, O., Fontanges, M., Schiano, A., Rauch, F., Gonnet, J-M. (2003). Recommandations pour la pra - tique clinique. Prise en charge des autres ecto - pies annexielles (cornuale, interstitielle, angu laire, ovarienne). J Gynecol Ostet Reprod, 32 (suppl.):3S93-3S100. 